Pontifícia $U_{\text {Niversidade }} C_{\text {atólica }}$

GRAVB:

Guilherme Barros de Castro Filho

Estudo do fluxo e transporte de gasolina pura e misturada com etanol em meios porosos não saturados

Dissertação apresentada ao Programa de Pós-Graduação em Engenharia Civil da PUC-Rio como requisito parcial para obtenção do título de Mestre em Engenharia Civil.

Orientadores: Eurípedes do Amaral Vargas Júnior Patrícia Österreicher-Cunha 


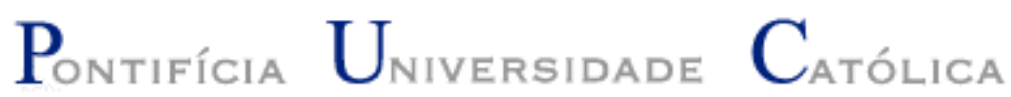

\section{Estudo do fluxo e transporte de gasolina pura e misturada com etanol em meios porosos não saturados}

\begin{abstract}
Dissertação apresentada como requisito parcial para obtenção do grau de Mestre pelo Programa de Pós-Graduação em Engenharia Civil da PUC-Rio. Aprovada pela Comissão Examinadora abaixo assinada.
\end{abstract}

\author{
Eurípedes do Amaral Vargas Jr. \\ Orientador \\ PUC - Rio
}

Patrícia Österreicher-Cunha

Co-orientadora

PUC - Rio

José Tavares Araruna Júnior PUC - Rio

Elisabeth Ritter

UERJ

\section{Everton de Oliveira} HIDROPLAN

José Eugênio Leal

Coordenador(a) Setorial do Centro Técnico Científico - PUC-Rio 
Todos os direitos reservados. É proibida a reprodução total ou parcial do trabalho sem autorização da universidade, do autor e do orientador.

\section{Guilherme Barros de Castro Filho}

Graduou-se em Engenharia Civil pela Pontifícia Universidade Católica do Rio de Janeiro em 2004. As principais áreas de interesse e linhas de pesquisa são: mecânica dos solos, geotecnia ambiental e experimental.

Ficha Catalográfica

Castro Filho, Guilherme Barros

Estudo do fluxo e transporte de gasolina pura e misturada com etanol em meios porosos não saturados/Guilherme Barros de Castro Filho; orientador: Eurípedes do Amaral Vargas Júnior; co-orientadora: Patrícia Österreicher-Cunha. - Rio de Janeiro: PUC, Departamento de Engenharia Civil, 2007.

155 f.; $30 \mathrm{~cm}$

Dissertação (Mestrado) - Pontifícia Universidade Católica do Rio de Janeiro, Departamento de Engenharia Civil.

Inclui referências bibliográficas.

1. Engenharia Civil - Teses. 2. Gasolina 3. Modelo HSSM 4. Zona vadosa 5. Ensaio de coluna. I. Eurípedes do Amaral Vargas Júnior. II. Patrícia Österreicher III. Pontifícia Universidade Católica do Rio de Janeiro. Departamento de Engenharia Civil. IV. Título 


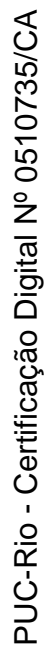

À minha família. 


\section{Agradecimentos}

Ao meu orientador de Tese de Mestrado, Eurípedes do Amaral Vargas Júnior, pela orientação e amizade sempre demonstrada.

À minha co-orientadora de Tese de Mestrado Patrícia Österreicher Cunha, que sempre esteve disponível para qualquer ajuda.

Ao órgão CNPq pelo apoio financeiro e à PUC-Rio que me deu a oportunidade de fazer o mestrado.

A todos os colegas da Pós-graduação em Engenharia civil, pela amizade e companheirismo.

Aos funcionários William, Amauri, José Raimundo e Josué, pela amizade e assistência dispensada no Laboratório de Geotecnia da Pontifícia Universidade Católica do Rio de Janeiro.

À Mónica, que sempre me auxiliou na execução de ensaios no Laboratório de Geotecnia da PUC-Rio.

À Raquel Velloso pela atenção e ajuda na solução de problemas relacionados com o presente estudo.

À aluna de Iniciação Científica, Carina Rennó Siniscalchi, pela participação e auxílio na realização dos ensaios no laboratório.

A todos os professores e colegas de turma de pós-graduação da PUC-Rio, onde também me graduei, por suas importantes participações na minha formação profissional.

À minha família, que sempre me apoiou e estimulou a estudar e buscar desafios para a minha vida.

À pessoa maravilhosa que conheci no início do curso de mestrado, minha namorada Viviana, pela companhia e amor dedicado ao longo destes anos. Você foi fundamental para eu persistir e concluir a tese. 


\section{Resumo}

Castro Filho, Guilherme Barros. Estudo do fluxo e transporte de gasolina pura e misturada com etanol em meios porosos não saturados. Rio de Janeiro, 2007. 155 p. Dissertação de Mestrado Departamento de Engenharia Civil, Pontifícia Universidade Católica do Rio de Janeiro.

O derramamento no solo de poluentes imiscíveis com a água (NAPLs) devido a vazamentos de tanques de armazenamento ou dutos e acidentes de transporte é de grande interesse, pois o NAPL e/ou seus constituintes podem migrar através da zona vadosa até atingir o lençol freático e contaminar as fontes de água potável. No Brasil, a maioria dos postos de combustível e terminais de armazenamento possui tanques de etanol, gasolina misturada com etanol e óleo diesel. Um eventual derramamento ou vazamento de hidrocarbonetos derivados de petróleo, conhecidos como LNAPL, misturados com etanol tem um maior potencial de contaminação em função do efeito de co-solvência.

A motivação deste trabalho é que muitos estudos têm sido realizados com principal interesse na zona saturada e pouco se sabe sobre o comportamento destes contaminantes na zona não saturada (McDowell e Powers, 2003 e Österreicher et al., 2007).

Portanto, foram realizados ensaios de coluna com esferas de vidro com o objetivo de simular os vazamentos de hidrocarbonetos líquidos na superfície e comparar a massa de benzeno que fica retida no meio poroso em um vazamento de gasolina pura ou com etanol. Para avaliar estes ensaios em uma dimensão foi utilizado um programa disponível no sítio da Agência de Proteção Ambiental dos Estados Unidos (EPA) chamado Hydrocarbon Spill Screening Model (Weaver et al., 1994) e implementado um modelo para efetuar o balanço de massa e considerar o efeito de co-solvência em função da adição de etanol na gasolina comercial brasileira.

Os resultados dos ensaios de laboratório foram comparados com os obtidos através do modelo supracitado e apresentaram uma aproximação satisfatória da previsão do comportamento do contaminante.

\section{Palavras-chave}

Gasolina; ensaio de coluna; zona vadosa; LNAPL; solo não saturado; Modelo HSSM. 


\section{Abstract}

Castro Filho, Guilherme Barros. Flux and transport study of pure gasoline and gasoline blended ethanol in unsaturated porous media. Rio de Janeiro, 2007. 155 p. Msc. Thesis - Civil Engineering Department, Pontifícia Universidade Católica do Rio de Janeiro.

The subsurface release of water immiscible pollutants (NAPLs) due to leaks in storage tanks or pipelines and spilling transportation accidents is of great concern, since the NAPL or its constituents may migrate through the vadose zone until reaching the water table and eventually contaminate clean water sources. In Brazil, most of the gas stations store ethanol, pure and gasoline blended ethanol, diesel, among others in tanks. A spill or leak of petroleum hydrocarbons, known as LNAPL (light Nonaqueous phase liquid), combined with ethanol has a major contamination potential associated to cosolvency effects.

The motivation of this work is that many studies have been developed with special interest in the saturated zone and little is known about the behavior of these pollutants in the vadose zone (McDowell e Powers, 2003 e Österreicher et al., 2007).

Thus, one dimensional column tests were performed in a glass porous media to simulate the spill of hydrocarbons in the subsurface and compare the retained mass of benzene in the porous media after a release of a pure or ethanol blended gasoline. In order to evaluate these 1D tests results, the Hydrocarbon Spill Screening Model - HSSM (Weaver et al., 1994) was used. This program can be found in the Environmental Protection Agency (EPA) website. A model was also implemented to consider the cosolvency effects in function of the ethanol addition in Brazilian's commercial gasoline.

The laboratory's results were compared with the ones obtained by the model mentioned above and showed a satisfactory approximation for the prediction of the contaminant behavior.

\section{Key Words}

Gasoline; column tests; vadose zone; LNAPL, unsaturated soil; HSSM model. 


\section{Lista de símbolos e abreviações}

$\% \mathrm{P}_{\mathrm{i}}=$ Porcentagens em peso do líquido i (água, gasolina e etanol);

$\mathrm{A}_{\text {COLUNA }}=$ Área transversal da coluna;

$\mathrm{A}_{\text {contaminada }}=$ área contaminada ou área da seção transversal da coluna de ensaio;

BTEX = Benzeno, Tolueno, Etilbenzeno e Xileno;

$\mathrm{C}_{\text {BENZ_NAPL }}^{\prime}=$ Concentração de benzeno na gasolina após dissolução deste na água;

$\mathrm{C}_{\mathrm{B}, \mathrm{GAS}}=$ Solubilidade do constituinte presente em uma mistura;

$\mathrm{C}_{\mathrm{BENZ} \_ \text {NAPL }}=$ Concentração de benzeno na gasolina;

$\mathrm{CC}=$ coeficientes de curvatura;

$\mathrm{cm}=$ Centímetro;

$\mathrm{C}_{\mathrm{m}}=$ Solubilidade do soluto na mistura água - co-solvente;

$\mathrm{CNU}=$ coeficientes de não uniformidade;

$\mathrm{Cp}=$ Centipoise

$\mathrm{C}_{\mathrm{s}}=$ Concentração no solo;

cte. $=$ constante;

$\mathrm{C}_{\mathrm{w}}=$ Concentração em uma fase em termos da concentração na água;

$\mathrm{C}_{\mathrm{w}}=$ Concentração na fase aquosa;

$\mathrm{C}_{\mathrm{wk}}=$ Solubilidade de um constituinte químico na água;

$\mathrm{dL}_{\mathrm{f}}=$ Derivada total da posição da frente de infiltração;

DNAPL $=$ Dense Nonaqueous Phase Liquid

$\mathrm{dt}=$ Derivada total do tempo;

e = índice de vazios;

e = Subscrito indicando etanol;

$E_{F . A .}=$ Teor de etanol na Fase aquosa;

$E_{F . O .}=$ Teor de etanol na fase orgânica; 
EPA = Environmental Protect Agency;

$\mathrm{f}_{\mathrm{c}}=$ Fração de volume de co-solvente na fase aquosa;

F.A. = Fase Aquosa;

F.O. = Fase Orgânica;

$f_{B E N Z \_N A P L}=$ Fração mássica de constituinte (benzeno) presente na fase NAPL;

$\mathrm{f}_{\mathrm{oc}}=$ Fração de carbono orgânico no meio;

$\mathrm{G}_{\mathrm{s}}=$ densidade dos grãos;

$\mathrm{h}_{\mathrm{ce}}=$ Pressão de entrada de ar;

$\mathrm{H}_{\text {COLUNA }}=$ Altura da coluna;

$\mathrm{H}_{\mathrm{i}}=$ Carga constante inicial de NAPL;

HSSM = Hydrocarbon Spill Screening Model

i = Líquidos: água, etanol e gasolina;

$\mathrm{K}=$ Permeabilidade efetiva do NAPL;

$\mathrm{K}_{\mathrm{d}}=$ Coeficiente de partição solo-água de uma fase;

$\mathrm{K}_{\mathrm{H}}=$ Coeficiente de partição ar-água de uma fase;

$\mathrm{K}_{H}{ }^{\prime}=$ Constante de Henry adimensional;

$\mathrm{K}_{\mathrm{o}}=$ Coeficiente de partição NAPL-água de uma fase;

$\mathrm{K}_{\mathrm{oc}}=$ Coeficiente de partição de carbono orgânico;

$\mathrm{K}_{\mathrm{os}}=$ Permeabilidade saturada do NAPL;

$\mathrm{K}_{\mathrm{ow}}=$ Coeficiente de partição octanol/água;

$\mathrm{kPa}=$ Kilo Pascal;

$\mathrm{k}_{\mathrm{r}}=$ Permeabilidade relativa do NAPL;

$\mathrm{k}_{\mathrm{rom}}=$ Permeabilidade relativa máxima do NAPL;

$\mathrm{K}_{\mathrm{ws}}=$ Permeabilidade saturada a água;

$L_{f}=$ posição da frente de infiltração;

$\mathrm{L}_{\mathrm{fc}}=$ Posição da frente de saturação para o tempo $t_{c}$;

LNAPL = Light Nonaqueous Phase Liquid;

$L_{\text {plateau }}=$ Posição da frente de saturação para o $t_{\text {plateau }}$; 
$L_{\text {pond }}=$ Posição da frente de saturação para o $t_{\text {pond; }}$

$\mathrm{m}=$ Metro;

$\mathrm{M}_{\text {BENZ_DISSOLV. }}^{\prime}=$ Massa de benzeno dissolvida que permaneceu na coluna;

$\mathrm{M}_{\mathrm{BENZ} \_ \text {NAPL }}^{\prime}=$ Massa de benzeno na gasolina retida na coluna;

$\mathrm{M}_{\text {BENZ_RETIDA }}^{\prime}=$ Massa de benzeno retida na coluna;

MEEN_DISSOLV = Massa de benzeno dissolvida em um volume de água inicial;

$M_{\text {BENZ_F.A. }}=$ Massa de benzeno dissolvida que saiu na fase aquosa drenada da coluna;

MEEN_INFILT = Massa de benzeno contida na gasolina infiltrada;

$M_{B E N Z \_N A P L}=$ Massa de benzeno retida na coluna na fase NAPL;

$M_{\text {BENZ_RETIDA }}=$ Massa de constituinte retida na coluna;

MBENZENO ADICIONADA = Massa de benzendo adicionada;

MBENZENO DRENADA = Massa de benzendo drenada;

$M_{\text {BENZENO RETIDA }}=$ Massa de benzeno retida;

$\mathrm{Mg} / \mathrm{m}^{3}$ = Mega grama por metro cúbico;

$\mathrm{M}_{\mathrm{i}}=$ Massa de líquido i no volume unitário do meio poroso;

$\mathrm{mm}=$ Milímetro;

$\mathrm{M}_{\text {NAPL_RETIDA }}=$ Massa de NAPL retida na coluna;

$M_{t}=$ Massa total de líquidos no volume unitário do meio poroso;

NAPL = Nonaqueous Phase Liquid;

$\mathrm{NBR}=$ Norma Brasileira;

0 = Subscrito indicando NAPL ou gasolina;

PUC-Rio = Pontifícia Universidade Católica do Rio de Janeiro;

$P_{\mathrm{vp}}=$ Pressão de vapor;

$q_{0}=$ Velocidade de infiltração;

$\mathrm{R}=$ Constante universal do gás;

RETC = Retention Curve Model (van Genuchten et al., 1991);

S = Segundo; 
$S_{w}^{\prime}=$ Saturação de água que permaneceu na coluna após a infiltração da gasolina com etanol;

$S^{\prime}{ }_{w}=$ saturação de água retida no meio poroso;

$\mathrm{S}_{\text {w_DREN }}=$ Redução na saturação de água na coluna após a infiltração da gasolina com etanol;

$\mathrm{S}_{\mathrm{a}}=$ Saturação inicial de ar;

$\mathrm{S}_{\mathrm{ar}}=$ Saturação residual de ar;

$\mathrm{S}_{\mathrm{GAS}}=$ Saturação de gasolina pura retida no meio poroso;

$\mathrm{S}_{\mathrm{i}}=$ Saturação de líquido i no volume unitário do meio poroso;

$\mathrm{S}_{\mathrm{k}}=$ Solubilidade do constituinte puro na água;

$\mathrm{S}_{\mathrm{o}}=$ Saturação de NAPL;

$\mathrm{S}_{\mathrm{om}}=$ Saturação máxima de NAPL;

$\mathrm{S}_{\mathrm{or}}=$ Saturação residual de NAPL;

$\mathrm{SP}=$ Sigla do SUCS para denominar uma areia mal graduada ou S (Sand) $\mathrm{P}$ (Poorly graded);

$\mathrm{S}_{\text {TOTAL }}=$ Saturação total de líquidos retida no meio poroso;

SUCS = Sistema Unificado de Classificação dos Solos;

$\mathrm{S}_{\mathrm{w}}=$ Saturação inicial de água na coluna;

$\mathrm{S}_{\mathrm{w}}=$ saturação média de água no meio poroso;

T = Temperatura em Kelvin;

$\mathrm{t}=$ tempo

$t_{c}=$ Tempo de duração de carga constante de NAPL;

$t_{\text {plateau }}=$ Instante em a saturação da frente de avanço do NAPL deixa de ser máxima;

$t_{\text {pond }}=$ Instante em que cessa a carga de NAPL na superfície;

$\mathrm{v}=$ Volume molar;

$\mathrm{V}_{\text {ÁGUA F.O. }}=$ Volume de água drenado na fase orgânica;

$\mathrm{V}_{\mathrm{w}}=$ Volume de água na coluna após a infiltração da gasolina com etanol;

$V_{\text {ÁGUA DREN TOTAL }}=$ Volume de água drenado total; 
$\mathrm{V}_{\text {ÁGUA F.A. }}=$ Volume de água drenado na fase aquosa;

$\mathrm{V}_{\text {água inicial }}=$ Volume de água inicial na coluna;

$\mathrm{V}_{\text {COLUNA }}=$ Volume do meio poroso na coluna;

$\mathrm{V}_{\mathrm{e}}=$ Volume de etanol no volume unitário do meio poroso;

$V_{\text {EFLUENTE }}=$ Volume de efluente coletado na proveta ao fim do ensaio;

$\mathrm{V}_{\mathrm{F} . \mathrm{A} .}=$ Volume da fase aquosa;

$\mathrm{V}_{\text {FASE_AQUOSA }}=$ Volume de fase aquosa drenada;

$\mathrm{V}_{\text {FASE_ORGÂNICA }}=$ Volume da fase orgânica drenada;

$\mathrm{V}_{\mathrm{GAS}}=$ Volume de gasolina injetado;

$\mathrm{V}_{\text {GAS DRENADO }}=$ Volume de gasolina drenado;

$\mathrm{V}_{\mathrm{GAS} \text { RETIDO }}=$ Volume de gasolina retido;

$V_{i}=$ Volume de líquido i no volume unitário do meio poroso;

$\mathrm{v}_{\mathrm{i}}=$ Volumes molares parciais dos constituintes;

$\mathrm{V}_{\text {material total }}=$ Volume total de material granular;

$\mathrm{V}_{\text {NAPL_INFILT }}=$ Volume de NAPL infiltrado;

$\mathrm{V}_{\text {NAPL_RETIDO }}=$ Volume de NAPL retido na coluna;

$\mathrm{V}_{\text {SEGMENTO }}=$ Volume dos segmentos de material poroso;

$V_{t}=$ Volume total unitário de meio poroso;

$V_{\text {total }}=$ Volume total de material contaminado até a profundidade $L_{\text {pond; }}$;

$\mathrm{V}_{\mathrm{w}}=$ Volume de água inicial na coluna;

$\mathrm{V}_{\mathrm{w}_{\text {_DREN }}}=$ Volume de água que foi drenado após a infiltração da gasolina com etanol;

w = Subscritos indicando água;

$\mathrm{W}_{\text {ÁGUA F.A }}=$ Teor de água na fase aquosa;

W $_{\text {ÁGUA F.O }}=$ Teor de água na fase orgânica;

$\mathrm{W}_{\mathrm{F} . \mathrm{A} .}=$ Teor de água na fase aquosa;

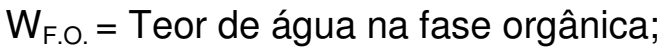

$\mathrm{w}_{\mathrm{i}}=$ Peso molecular do constituinte $\mathrm{i} ;$ 
$\mathrm{X}=$ Fração molar de etanol na fase aquosa;

$\mathrm{X}_{\mathrm{i}}=$ Fração molar do constituinte $\mathrm{i}$;

$X_{k}=$ Fração molar do constituinte na fase NAPL;

$\mathrm{z}=$ eixo vertical para baixo representando a profundidade;

$\Delta \mathrm{V}_{\text {NAPL_RETIDO }}=$ Volume de NAPL retido no segmento;

$\Delta \mathrm{z}=$ Altura do segmento de material poroso;

$\Delta \theta=$ Incremento de umidade volumétrica de NAPL;

$\Delta \rho_{\mathrm{ao}}=$ Diferença entre as densidades do ar e da água;

$\gamma_{d}=$ peso específico seco;

$\gamma_{k}=$ Coeficiente de atividade do constituinte na fase NAPL;

$\lambda=$ Índice de distribuição dos tamanhos dos poros;

$\mu=$ Viscosidade do NAPL ou gasolina;

$\theta_{\mathrm{i}}=$ Umidade volumétrica do líquido i no volume unitário do meio poroso;

$\theta_{0}=$ Umidade volumétrica de NAPL;

$\theta r=$ Umidade volumétrica residual;

$\theta s$ = Umidade volumétrica saturada;

$\rho_{\mathrm{e}}=$ Densidade do etanol;

$\rho_{\mathrm{i}}=$ Densidade do líquido i;

$\rho_{o}=$ Densidade do NAPL ou gasolina;

$\rho_{\mathrm{w}}=$ Densidade da água;

$\sigma_{\mathrm{ao}}=$ Tensão interfacial ar-NAPL;

$\sigma_{\mathrm{aw}}=$ Tensão interfacial ar-água;

$\psi=$ carga de pressão;

$\psi_{\mathrm{a}}=$ Sucção inicial;

$\psi_{\text {baw }}=$ Pressão de entrada de ar em um sistema ar-água;

$\psi_{f_{0}}=$ Sucção na frente de saturação; 


\section{Sumário}

1 Introdução 22

2 Revisão Bibliográfica 26

2.1 Vazamentos e derramamentos 26

2.2 Fluxo e Distribuição de NAPLs no Solo 30

$\begin{array}{lll}2.2 .1 & \text { Fluxo de DNAPLS } & 31\end{array}$

2.2.2 Fluxo de LNAPLS 33

2.3 Conceitos de Fluxo Multifásico 35

2.3.1 Tensões superficial e interfacial $\quad 35$

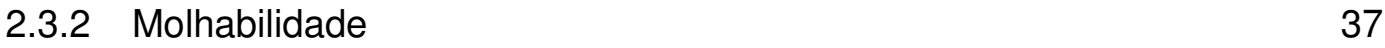

$\begin{array}{lll}\text { 2.3.3 } & \text { Pressão capilar } & 39\end{array}$

2.3.4 Curva característica $\quad 42$

2.3.5 Condutividade hidráulica 46

2.3.6 Saturação residual $\quad 49$

2.4 Partição de Solutos $\quad 52$

2.4.1 Misturas de fluidos $\quad 52$

2.4.2 Equilíbrio multifásico $\quad 54$

2.4.3 Relação de partição linear de solutos 55

2.5 Co-solvência $\quad 57$

2.5.1 Diagrama ternário de fases 62

2.5.2 Efeito da adição de etanol à gasolina no fluxo multifásico 65

3 Modelos para o estudo do fluxo e transporte de gasolina pura e misturada com etanol na zona vadosa $\quad 70$

$\begin{array}{lll}3.1 & \text { Introdução } & 70\end{array}$

$\begin{array}{lll}3.2 & \text { Modelo HSSM } & 71\end{array}$

3.3 Implementação de um modelo para gasolina com etanol 87

4 Ensaios de laboratório 93

4.1 Caracterização física do material granular 94

4.1.1 Curva granulométrica 94

$\begin{array}{lll}\text { 4.1.2 Densidade dos grãos } & 95\end{array}$ 
4.1.3 Compacidade 95

4.2 Curva característica 96

$\begin{array}{lll}\text { 4.3 Densidade e viscosidade dos fluidos } & 99\end{array}$

4.4 Ensaios de coluna $\quad 99$

4.4.1 Metodologia dos ensaios de coluna 100

4.4.2 Procedimentos e metodologias das análises químicas 110

5 Apresentação e discussão dos resultados 112

5.1 Introdução 112

5.2 Resultados dos ensaios de coluna 112

6 Conclusões e Sugestões 143

6.1 Conclusões 143

$\begin{array}{lll}6.2 & \text { Sugestões } & 145\end{array}$

$\begin{array}{ll}\text { Referências Bibliográficas } & 146\end{array}$ 


\section{Lista de figuras}

Figura 2.1: Figura esquemática de derramamentos devido a acidentes com caminhão (Carrillo, 2000).

Figura 2.2-Vazamentos de gasolina na bomba de abastecimento (CETESB, 2003). 28

Figura 2.3-Detalhe de corrosão em tanque metálico (CETESB, 2003). 29 Figura 2.4 - Modelo conceitual do comportamento do DNAPL no meio poroso (adaptado de Newel et al., 1995).

Figura 2.5- Modelo conceitual do comportamento do LNAPL no meio poroso (adaptado de Mercer and Cohen, 1990).

Figura 2.6- Forças de atração entre moléculas no interior e na superfície de um líquido (Shaw, 1998 apud Borges, 2002 ).

Figura 2.7- Tensões interfacial entre dois fluidos e uma superfície (Sahimi, 1994)

Figura 2.8- Algumas situações de molhagem (Carrillo, 2000).

Figura 2.9- Altura de ascensão e pressão da água em um tubo capilar (Pinto, 2002).

Figura 2.10- Curvas característica típicas para areia e argila (adaptado de Charbeneau, 2000).

Figura 2.11- Variação da pressão capilar em função do grau de saturação (adaptado de Fetter, 1993).

Figura 2.12- Curva típica de permeabilidade relativa. O subscrito w se refere ao fluido molhante e nw, ao fluido não molhante (Bear, 1972)

Figura 2.13- Retenção do fluido não molhante pelo processo de snap-off (Chatzis et al., 1983 apud Ferreira, 2003).

Figura 2.14- Retenção do fluido não molhante pelo processo de bypassing (Chatzis et al., 1983 apud Ferreira, 2003). 51

Figura 2.15- Partição em um sistema multifásico (Charbeneau, 2000). 55 Figura 2.16- Log da solubilidade dos constituintes BTX em função da fração de volume de etanol (Corseuil et al., 1999).

Figura 2.17 - Concentração aquosa dos BTX (mg/L), em função da fração de volume de etanol no sistema gasolina-etanol-água (Corseuil e $\begin{array}{ll}\text { Fernandes, 1999). } & 61\end{array}$

Figura 2.18- Diagrama ternário de fases (Borges, 2002). 63 
Figura 2.19- Diagrama ternário para água, etanol e gasolina com os valores de tensão interfacial em mN/m (Bicalho, 1997).

Figura 2.20- Tensão interfacial de diferentes gasolinas e constituintes orgânicos com água na presença de etanol (Kowles e Powers, 1997, exceto onde especificado).

Figura 2.21- Tensão superficial da água com o ar e da água em equilíbrio com uma fase orgânica (Kowles e Powers, 1997, exceto onde especificado).

Figura 2.22- Tensão interfacial entre as fases gasolina e aquosa versus a fração molar de etanol na fase aquosa (Bicalho, 1997).

Figura 3.1- Apresentação esquemática do funcionamento de cada módulo (adaptado de Weaver, 1994).

72

Figura 3.2- Comparação esquemática entre uma frente abrupta e difusiva (adaptado de Weaver et al., 1994).

74

Figura 3.3- Comparação entre os dados medidos de um ensaio de coluna e os dados previstos pelo programa HSSM (adaptado de Weaver et al., 1994). 75

Figura 3.4- Visualização esquemática do modelo de infiltração de Green-Ampt (adaptado de Charbeneau, 2000).

Figura 3.5- Visão esquemática dos quatro períodos principais do modelo de infiltração do NAPL (adaptado de Charbeneau, 2000).

82

Figura 3.6- Visualização do procedimento de cálculo das massas de NAPL e constituinte retidas na coluna após a drenagem do contaminante.

86

Figura 3.7- Diagrama ternário de fases esquemático para o exemplo de cálculo apresentado.

89

Figura 4.1-Curva granulométrica das esferas de vidro.

94

Figura 4.2-Equipamento de Placa de Pressão (Carrillo, 1994 apud Carrilo, 2000)

96

Figura 4.3-Curva característica de 4 amostras e ajuste dos dados com o modelo de Brooks e Corey. $\quad 98$

Figura 4.4- Saturação de água retida na coluna após longo período de drenagem.

Figura 4.5- Aplicação de sucção na base da coluna para reduzir a saturação da camada inferior.

102

Figura 4.6- Apresentação da coluna de ensaio e da malha colocada na base para sustentar o material.

102

Figura 4.7- Camada de material na base com maior saturação.

Figura 4.8- Coluna em contato com areia seca para drenar a camada inferior de 
material.

Figura 4.9- Permeâmetro desenvolvido na PUC-Rio para aplicação de carga constante de contaminante.

Figura 4.10- Esquema do ensaio de coluna com injeção de gasolina. 106

Figura 4.11- Separação das fases aquosa (amarelo) e orgânica (vermelho). 107

Figura 4.12- Efluente dos ensaios de gasolina com etanol sem corante apresentando duas fases.

108

Figura 4.13- Seqüência (1-9) do avanço da frente de saturação de gasolina nos ensaios de coluna ao longo do tempo.

109

Figura 4.14- Frascos selados contendo amostras da gasolina pura injetada e drenada.

Figura 4.15- Cromatograma ilustrativo dos resultados obtidos através da análise química.

Figura 5.1- Volumes medidos nos ensaios de gasolina pura.

Figura 5.2- Volumes medidos nos ensaios de gasolina com etanol.

Figura 5.3- Massa retida dos constituintes BTEX no meio poroso nos 2 ensaios com gasolina pura e a média.

Figura 5.4- Concentração média dos constituintes BTEX nas fases orgânica e aquosa no efluente dos quatro ensaios de coluna.

Figura 5.5- Concentração média de água e etanol nas fases orgânica e aquosa no efluente dos quatro ensaios de coluna.

Figura 5.6- Concentração média total dos constituintes BTEX no efluente dos 4 ensaios.

Figura 5.7- Massas retidas dos constituintes BTEX e etanol nos ensaios com gasolina contendo etanol.

Figura 5.8- Avanço da frente de saturação de gasolina pura e com etanol nos ensaios de coluna em laboratório.

Figura 5.9- Concentração média de BTEX na gasolina pura e com etanol injetada.

Figura 5.10- Concentrações médias dos constituintes BTEX nos efluentes dos ensaios de gasolina pura e com etanol.

Figura 5.11- Comparação entre as porcentagens médias de BTEX retidas nos ensaios.

Figura 5.12- Avanço da frente de saturação dos ensaios de coluna e estimado através do modelo de infiltração para gasolina pura.

Figura 5.13- Análise de sensibilidade do parâmetro viscosidade na massa de 
benzeno retida.

Figura 5.14- Análise de sensibilidade do parâmetro tensão interfacial ar-gasolina na massa de benzeno retida.

136

Figura 5.15- Análise de sensibilidade do parâmetro saturação residual de gasolina na massa de benzeno retida.

Figura 5.16- Análise de sensibilidade do parâmetro tensão interfacial ar-água na massa de benzeno retida.

138

Figura 5.17- Avanço da frente de saturação dos ensaios de coluna e estimado através do modelo de infiltração para gasolina com etanol. 


\section{Lista de tabelas}

Tabela 2.1 - Composições dos pontos A, B e C obtidas a partir do diagrama ternário.

Tabela 2.2- Parâmetros das gasolinas usadas no trabalho de Finotti (2003). 68

Tabela 4.1- Resumo das características granulométricas das esferas de vidro. 95 Tabela 4.2-Parâmetros obtidos com o ajuste dos pontos utilizando o programa RETC.

Tabela 5.1- Volume total de material granular e volume de água inicial na coluna.

Tabela 5.2- Índices físicos do meio poroso determinados para cada ensaio de coluna.

Tabela 5.3- Volumes medidos dos efluentes coletados na proveta.

Tabela 5.4- Teores de água e etanol nas fases aquosa e orgânica drenadas. 115 Tabela 5.5- Volume de água drenado na fase aquosa (F.A.), orgânica (F.O.) e total.

Tabela 5.6- Saturação de água, gasolina e total de líquidos retidos na coluna ao final dos ensaios.

Tabela 5.7- Fração e massa de BTEX na gasolina pura injetada e no efluente.

Tabela 5.8- Fração e massa retida de BTEX na coluna nos ensaios com gasolina pura.

Tabela 5.9- Concentração de BTEX e etanol na gasolina injetada. 120

Tabela 5.10- Concentração de BTEX, etanol e água na fase orgânica. 120

Tabela 5.11- Concentração de BTEX, etanol e água na fase aquosa.

Tabela 5.12- Concentração total no efluente constituído das fases aquosa e orgânica. 124

Tabela 5.13- Concentrações retidas de BTEX e etanol nos 4 ensaios. 125 Tabela 5.14- Parâmetros utilizados no modelo de simulação de infiltração de gasolina pura.

Tabela 5.15- Resultados do balanço de massa dos ensaios de coluna com gasolina pura e do modelo de infiltração.

Tabela 5.16- Porcentagem em relação ao volume e a massa que foi injetada na coluna para os ensaios de coluna em laboratório e simulado com o modelo. 134 Tabela 5.17- Parâmetros utilizados no modelo de simulação de infiltração de 
gasolina com etanol.

Tabela 5.18- Resultados do balanço de massa dos ensaios de coluna com gasolina com etanol e do modelo de infiltração.

Tabela 5.19- Porcentagem em relação ao volume e a massa que foi injetada na coluna para os ensaios de coluna em laboratório e simulado com o modelo. 141 Tabela 5.20- Comparação entre os resultados do balanço de massa estimado pelas análises químicas para os ensaios com gasolina pura e com etanol. 142 Tabela 5.21- Comparação entre os resultados do balanço de massa estimado através da simulação com o modelo dos ensaios com gasolina pura e com etanol. 